\title{
The preparation of the quasi-Bell-base of entangled squeezed states via atom-cavity-field Raman interaction
}

\author{
Xinhua Cai \\ College of Physics and Electronics, Hunan University of Arts and Science, Changde 415000, China \\ xhcai@sina.com
}

Keywords: Quantum Information, Quasi Bell Base, Squeezed Vacuum States, $\Lambda$-Type Three-Level Atom, Cavity Quantum Electrodynamics.

Abstract. In quantum communication and quantum computing, the Bell state acts as an important role. For two qubit of discrete variable, Bell state is composted by two orthogonal to each other's physical state of a physical system. For the physical system consisting of continuous variables, a scheme is presented for generating the quasi-Bell-states of entangled squeezed states by using the Raman interaction of an $\Lambda$-type three-level atom with squeezed vacuum states (SVS) cavity fields. In this scheme, an atom initially prepared in its ground state is injected in proper order into several cavities initially prepared each in the SVS. After the atom interacting with these cavities, the entanglement of atom-cavity-field is generated. Afterwards, by measuring the atom we obtain the quasi-Bell-states of the entangled squeezed states. Also, the entanglement properties of bipartite entangled quasi-Bell-states as well as multipartite entangled SVSs are discussed.

\section{Introduction}

In recent years, entanglement has generated much interest in the quantum information processing such as quantum teleportation[1], superdense coding[2], quantum key distribution[3], telecoloning[4] and quantum positioning and clock synchronization[5]. Hence, quantum entanglement has been viewed as an essential resource for quantum information processing. The original study for quantum information processing focused on the systems with a finite-dimensional (discrete variable) state space, such as the polarizations of a photon or the discrete levels of an atom. Since the experimental demonstration of quantum teleportation of coherent states[6], much interest have arisen in continuous variable quantum information processing[7-8]. Thereupon, the entangled states with continuous variable receive much attention in the study of quantum entanglement[9-10].

In the case of two qubit of discrete variable, people like to introduce a set of four mutually orthogonal maximally entangled states as the base vectors, namely Bell-base, which may be denoted

$$
\left\{\begin{array}{l}
|\phi\rangle_{12}^{+}=\frac{1}{\sqrt{2}}\left(|00\rangle_{12}+|11\rangle_{12}\right) \\
|\phi\rangle_{12}^{-}=\frac{1}{\sqrt{2}}\left(|00\rangle_{12}-|11\rangle_{12}\right) \\
|\psi\rangle_{12}^{+}=\frac{1}{\sqrt{2}}\left(|01\rangle_{12}+|10\rangle_{12}\right) \\
|\psi\rangle_{12}^{-}=\frac{1}{\sqrt{2}}\left(|01\rangle_{12}-|10\rangle_{12}\right)
\end{array},\right.
$$

where 0 and 1 denote two mutually orthogonal states of a physical system, such as the polarizations of a photon or the discrete levels of an atom. However, for the continuous variable, there was a suggestion to implement a logical qubit encoding through treating a coherent superposition state, which can make a single mode continuous variable state as a qubit in two dimensional Hilbert space[11]. So similar base vectors to above have been defined by using two squeezed states with the same amplitude but a phase difference of $\pi|\xi\rangle$ and $|-\xi\rangle$, where $|\xi\rangle$ and $|-\xi\rangle$ are of nonorthogonal. Hence, these states are named as quasi-Bell-states. Some methods have been studied to generate the quasi-Bell-states such as the Kerr medium[12], cavity quantum electrodynamics[13-15]. In this paper, 
we present a scheme for generating quasi-Bell-states of entangled squeezed states via the Raman interaction of an $\Lambda$-type three-level atom with SVS cavity-field.

\section{Raman interaction}

First, let's briefly review the Raman interaction of atom-cavity-field. For a system consisting of a degenerate $\Lambda$-type three-level atom and a single mode field, the Hamiltonian is given by $(\hbar=1)$ [16]

$$
\begin{aligned}
\hat{H}= & \omega \widehat{a}^{+} \widehat{a}+\omega_{f}|f\rangle\langle f|+\omega_{0}(|e\rangle\langle e|+| g\rangle\langle g|)+g_{1}\left(\hat{a}^{+}|g\rangle\left\langle f\left|e^{-i \Delta t}+\widehat{a}\right| f\right\rangle\langle g| e^{i \Delta t}\right) \\
& +g_{2}\left(\widehat{a}^{+}|e\rangle\left\langle f\left|e^{-i \Delta t}+\widehat{a}\right| f\right\rangle\langle e| e^{i \Delta t}\right),
\end{aligned}
$$

where $|e\rangle$ and $|g\rangle$ are two degenerate lower of the atom, $|f\rangle$ is the upper level of the atom, $\hat{a}^{+}$and $\hat{a}$ are the bosonic creation and annihilation operators of the cavity field, g1(g2) is the coupling constant between the atomic transition $|f\rangle \rightarrow|g\rangle(|e\rangle)$ and cavity mode, $\omega_{0}$ and $\omega_{f}$ are the energies of the lower-level and upper-level of the atom, $\Delta$ is the detuning, respectively. If the atomic transition frequency is highly detuned from the cavity field frequency, the upper level $|f\rangle$ can be adiabatically eliminated. Under this condotion the effective Hamiltonian of this system is given by[17]

$$
\hat{H}_{e f f}=-\lambda \widehat{a}^{+} \widehat{a}(|e\rangle\langle g|+| g\rangle\langle e|)-\widehat{a}^{+} \widehat{a}\left(\beta_{1}|g\rangle\left\langle g\left|+\beta_{2}\right| e\right\rangle\langle e|\right),
$$

where $\lambda=g_{1} g_{2} / \Delta, \beta_{1}=g_{1}^{2} / \Delta, \beta_{2}=g_{2}^{2} / \Delta, \Delta=\left(\omega_{f}-\omega_{0}\right)-\omega$. The parameters $\beta_{1}$ and $\beta_{2}$ denote the intensity dependent Stark shifts of states $|g\rangle$ and $|e\rangle$, respectively. For convenience, let $g_{1}=g_{2}=g$, thus $\lambda=\beta_{1}=\beta_{2}=g^{2} / \Delta$.

Suppose that the atom is initially prepared in the ground state $|g\rangle$, and the cavity field is initially prepared in the SVS $|\xi\rangle$, the initial atom-cavity state is

$$
|\Psi(0)\rangle_{a-f}=|g\rangle \otimes|\xi\rangle,
$$

where $\xi=r e^{i \varphi}$ is any complex number with modulus $r$ and argument $\varphi$. An expansion in terms of Fock states is[18]

$$
|\xi\rangle=\sqrt{\operatorname{sech} r} \sum_{n=0}^{\infty} \frac{\sqrt{(2 n) !}}{n !}\left(-\frac{1}{2} e^{i \varphi} \tanh r\right)^{n}|2 n\rangle .
$$

In interaction picture, the time evolution of state vector of system is given by Schrödinger equation[19-20]

$$
i \frac{d|\Psi(t)\rangle}{d t}=\hat{H}_{e f f} \mid \Psi(t) .
$$

The state vector $|\Psi(t)\rangle$ is given by

$$
\begin{aligned}
|\Psi(t=\tau)\rangle & =\exp \left(-i \hat{H}_{e f f} t\right)|\Psi(0)\rangle_{a-f} \\
& =\frac{1}{2}\left[\left(|\xi\rangle+\left|\xi e^{4 i\left(g^{2} / \Delta\right) \tau}\right\rangle\right)|g\rangle-\left(|\xi\rangle-\left|\xi e^{4 i\left(g^{2} / \Delta\right) \tau}\right\rangle\right)|e\rangle\right] .
\end{aligned}
$$

where $\tau$ is the interaction time between the atom and cavity field, it can be controlled by adjusting the velocity of the atom. With the choice $\left(g^{2} / \Delta\right) \tau=\pi / 4$, we obtain

$$
|\Psi(\tau)\rangle_{a-f}=\frac{1}{2}[(|\xi\rangle+|-\xi\rangle)|g\rangle-(|\xi\rangle-|-\xi\rangle)|e\rangle] .
$$

Similarly, if the atom is initially prepared in its excited state $|e\rangle$, we can obtain

$$
|\Psi(\tau)\rangle_{a-f}=\frac{1}{2}[(|\xi\rangle+|-\xi\rangle)|e\rangle-(|\xi\rangle-|-\xi\rangle)|g\rangle] .
$$




\section{Preparation of quasi-Bell-states}

According to the description above, it is easy to realize the generation of the entangled SVSs. We assume that there are two cavities (referred to as 1 and 2), which are both initially prepared in the SVS $|\xi\rangle$, and that the atom is initially prepared in the ground state $|g\rangle$. Then the initial state of the whole system is given by

$$
|\Psi(0)\rangle_{a-f}=|g\rangle \otimes|\xi\rangle_{1} \otimes|\xi\rangle_{2} .
$$

Let atom enter cavity 1 and 2 in proper order with same interaction time $\tau$. After passage of the atom from these cavities the atom-field state is

$$
\left|\Psi^{\prime}\right\rangle_{a-f}=\frac{1}{2}\left(|\xi\rangle_{1}|\xi\rangle_{2}+|-\xi\rangle_{1}|-\psi\rangle_{2}\right)|g\rangle-\frac{1}{2}\left(|\xi\rangle_{1}|\xi\rangle_{2}-|-\xi\rangle_{1}|-\xi\rangle_{2}\right)|e\rangle .
$$

Thereupon, we perform an atom measurement. When the atom is detected in the ground state or excited state, the cavity field collapses correspondingly into

$$
\left|\Psi^{\prime \prime}\right\rangle_{f} \rightarrow|\xi\rangle_{1}|\xi\rangle_{2}+|-\xi\rangle_{1}|-\xi\rangle_{2}
$$

or

$$
\left|\Psi^{\prime \prime}\right\rangle_{f} \rightarrow|\xi\rangle_{1}|\xi\rangle_{2}-|-\xi\rangle_{1}|-\xi\rangle_{2} \text {. }
$$

After normalization we get two entangled SVSs

$$
\left|\phi^{ \pm}\right\rangle_{1,2}=\frac{1}{\sqrt{N_{ \pm}}}\left(|\xi\rangle_{1}|\xi\rangle_{2} \pm|-\xi\rangle_{1}|-\xi\rangle_{2}\right)
$$

where $N_{ \pm}=2\left(1 \pm k^{2}\right)$ are normalization constant and $k=\langle\xi \mid-\xi\rangle=\sqrt{\sec h(2 r)}$ is the overlap.

If the two cavity-fields are initially prepared in the SVSs with equal-amplitude and opposite-phase, the initial state of the whole system is given by

$$
|\Psi(0)\rangle_{a-f}=|g\rangle \otimes|\xi\rangle_{1} \otimes|-\xi\rangle_{2} \text {. }
$$

After the atom interaction with two cavity-fields the system evolves to

$$
\left|\Psi^{\prime}\right\rangle_{a-f}=\frac{1}{2}\left(|\xi\rangle_{1}|-\xi\rangle_{2}+|-\xi\rangle_{1}|\xi\rangle_{2}\right)|g\rangle-\frac{1}{2}\left(|\xi\rangle_{1}|-\xi\rangle_{2}-|-\xi\rangle_{1}|\xi\rangle_{2}\right)|e\rangle .
$$

By performing a similar measurement as above, we get two entangled SVSs

$$
\left|\psi^{ \pm}\right\rangle_{1,2}=\frac{1}{\sqrt{N_{ \pm}}}\left(|\xi\rangle_{1}|-\xi\rangle_{2} \pm|-\xi\rangle_{1}|\xi\rangle_{2}\right.
$$

Synthesizing Eq.(14) and Eq.(17), we obtain a set of quasi-Bell states based on SVSs in $2 \times 2$ Hilbert space. For bipartite entangled SVSs described by Eq.(14) and Eq.(17), they form a set of quasi-Bell basic states in $2 \times 2$ Hilbert space

$$
\left\{\begin{array}{l}
|\phi\rangle_{1,2}^{+}=\frac{1}{\sqrt{N_{+}}}\left(|\xi\rangle_{1}|\xi\rangle_{2}+|-\xi\rangle_{1}|-\xi\rangle_{2}\right) \\
|\phi\rangle_{1,2}^{-}=\frac{1}{\sqrt{N_{-}}}\left(|\xi\rangle_{1}|\xi\rangle_{2}-|-\xi\rangle_{1}|-\xi\rangle_{2}\right) \\
|\psi\rangle_{1,2}^{+}=\frac{1}{\sqrt{N_{+}}}\left(|\xi\rangle_{1}|-\xi\rangle_{2}+|-\xi\rangle_{1}|\xi\rangle_{2}\right) \\
|\psi\rangle_{1,2}^{-}=\frac{1}{\sqrt{N_{-}}}\left(|\xi\rangle_{1}|-\xi\rangle_{2}-|-\xi\rangle_{1}|\xi\rangle_{2}\right) .
\end{array} .\right.
$$

They are not orthogonal each other. In the limiting case of $r \rightarrow \infty$, the 4 entangled SVSs are orthogonal each other. Then these states reduce to standard Bell-states. Studies have shown that the entangled SVSs $|\phi\rangle^{-}$and $\left.|\psi\rangle\right\rangle^{-}$are maximally entangled state, their amount of entanglement are exactly one ebit and the entanglement is independent of the parameters involved. However, the states $|\phi\rangle^{+}$and $|\psi\rangle^{+}$are not maximally entangled state except in the case of $r \rightarrow \infty$. 


\section{Conclusions}

From what has been discussed above, we have presented a scheme to prepare bipartite entangled SVSs via the Raman interaction of a degenerate $\Lambda$-type three-level atom with cavity-fields in SVS. Analysis shows that, if initial cavity-field states are in two different SVSs $|\xi\rangle_{1}|\xi\rangle_{2}$ and $|\xi\rangle_{1}|-\xi\rangle_{2}$, we can obtain a set of quasi-Bell states $|\phi\rangle_{12}^{ \pm},|\psi\rangle_{12}^{ \pm}$based on SVSs in $2 \times 2$ Hilbert space. When the squeezed parameter $r \rightarrow \infty$, the two SVSs $|\xi\rangle$ and $|-\xi\rangle$ become orthogonal, and then these quasi-Bell states reduce to standard Bell-states. For multipartite SVS, it is possible to generate the entangled SVS with $N$ subsystems. We have proven that in the limits of $r \rightarrow \infty$ and $r \rightarrow 0$, the multipartite entangled SVS reduce correspondingly into GHZ state and W state.

\section{Acknowledgements}

This work was financially supported by the National Natural Science Foundation of China (Nos. 61144006, 61204104).

\section{References}

[1] C. H. Bennett, G.. Brassard and C. Crepeau, et al., Phys. Rev. Lett., Vol.70(1993), p.1895.

[2] C. H. Bennett and S. J. Wiesner, Phys. Rev. Lett., Vol.69(1992), p.2881.

[3] A. K. Ekert, Phys. Rev. Lett., Vol.67(1991), p.661.

[4] G. M. D'Ariano, Phys. Rev. Lett., Vol.76(1996), p.2832.

[5] V. Giovannetti, S. Lloyd, Maccone, Nature, Vol.412(2001), p.417.

[6] A. Furusawa, J. L. Sorensen, S. L. Braunstein, et al., Vol.282(1998), p.706.

[7] C. D. Ogden, M. Patemostro and M. S. Kim, Phys. Rev. A, Vol.75(2007),, 042325.

[8] D. Menzies and N. Korolkova, Phys. Rev A, Vol.74(2006), 042315.

[9] X. H. Cai, J. R. Guo and J. J. Nie, et al., Chin. Phys., Vol.15(2006), p.488.

[10] Y. B. Sheng, F. G. Deng and H. Y. Zhou, Phys. Rev. A, Vol.77(2008), 062325.

[11] P. T. Cochrane, G. J. Milburn and W. J. Munro, Phys. Rev. A, Vol. 59(1999), p.2631.

[12] B. Yurke and D. Stoler, Phys. Rev. Lett., Vol. 57(1986),p.13.

[13] Q. A. Turchette , C. J. Hood and W. Lange, et al., Phys. Rev. Lett., Vol. 75(1995),p. 4710.

[14] X. Li, Y. J. Zhang and Y. J. Xia, Comm. Theo. Phys., Vol.4(2008), p.467.

[15] S. B. Zheng, G. C. Guo, Physica A, Vol. 251(1998), P.507.

[16] X. H. Zhang, Z. Y. Yang and , P. P. Xu, Science in China Series G, Vol.52(2009), p. 1034.

[17] J. Recamier, O. Castaños, R. Jáuregui, and A. Frank, Phys. Rev. A, Vol. 61(2000), 063808.

[18] X. Zou and W. Mathis, Journal of Modern Optics, Vol. 52(2005), 2001.

[19] V. Giovannetti, D. Vitali, P. Tombesi, and A. Ekert, Phys. Rev. Vol.A, 62(2000), 032306.

[20] X. H. Cai and J. J. Nie, Applied Mechanics and Materials, Vol.571-572(2014), p.469. 